\title{
Offner imaging spectrometers
}

\section{Espectrómetros de imagen Offner}

\author{
H. González-Núñez ${ }^{1,2, S^{*}}$, R. de la Fuente ${ }^{1, S}$ \\ 1. Universidade de Santiago de Compostela, 15782 Galicia, Spain \\ 2. Pontificia Universidad Católica de Chile, 4860 Vicuña Mackenna, Santiago, Chile. \\ (*) E-mail: hegonzalez@uc.cl S: SEDOPTICA member
}

DOI: $10.7149 /$ OPA.50.1.49506

\begin{abstract}
:
A hyperspectral sensor or imaging spectrometer is a device that collects spatial and spectral information in hundreds of contiguous and closely spectral bands. Hyperspectral imagers have many applications in several areas such as agriculture, geology, oceanography, forestry, medicine, colorimetry, security, military and others. Most of the existing hyperspectral systems were developed considering satellite or high altitude flying aircrafts applications. However, the specific development of these technologies allows their use in many other less expensive applications. In this context, the work presented in this thesis allows building high quality and low cost Offner imaging spectrometers. Rapid and efficient methods were used for the design, assembly and characterization of this type of spectrometers. Two laboratory setups developed from analytical model-based designs have been assembled showing high spectral and spatial resolutions and low distortions without specific aberrations corrected gratings nor non-spherical elements.
\end{abstract}

Key words: Imaging spectrometry, Offner spectrometer, Optical design.

\section{RESUMEN:}

Un sensor hiperespectral o espectrómetro de imagen es un dispositivo que recoge información espacial y espectral en cientos de bandas contiguas y espectralmente estrechas. Los sensores hiperespectrales tienen muchas aplicaciones en diversas áreas como agricultura, geología, oceanografía, silvicultura, medicina, colorimetría, seguridad o aplicaciones militares entre otras. La mayor parte de los equipos hiperespectrales existentes fueron construidos pensando en aplicaciones desde satélites y/o aviones sobrevolando a gran altitud. No obstante, el desarrollo específico de estas tecnologías permite su uso en muchas otras aplicaciones con menor presupuesto. En este contexto, el trabajo presentado en esta de tesis doctoral permite la construcción de espectrómetros de imágenes Offner de alta calidad y bajo coste mediante métodos rápidos y eficientes de diseño, montaje y caracterización. Se han realizado dos montajes de laboratorio a partir de diseños basados en modelos analíticos mostrando unas altas resoluciones espectrales y espaciales y bajas distorsiones sin el uso de redes de difracción corregidas en aberraciones ni de elementos asféricos.

Palabras clave: Espectrometría de imagen, Espectrómetro Offner, Diseño óptico.

\section{REFERENCES AND LINKS / REFERENCIAS Y ENLACES}

[1] A. F. H. Goetz, "Three decades of hyperspectral remote sensing of the earth: A personal view," Remote Sens. Environ., 113, S5-S16 (2009). https://doi.org/10.1016/j.rse.2007.12.014

[2] A. F. H. Goetz, G. Vane, J. E. Solomon, B. N. Rock, "Imaging spectrometry for Earth remote sensing," Science, 228 (1985). https://doi.org/10.1126/science.228.4704.1147

[3] G. A. Shaw, H. K. Burke. Spectral imaging for remote sensing. Lincoln Lab. J., 14, 3-28 (2003). 
[4] J. Hill, J. Mégier, Imaging Spectrometry, a Tool for Environmental Observations. Kluwer Academic Dordrecht, Boston (1994). https://doi.org/10.1007/978-0-585-33173-7

[5] P. Mouroulis, R. O. Green, T. G. Chrien. "Design of pushbroom imaging spectrometers for optimum recovery of spectroscopic and spatial information," App. Opt., 39(13), 2210-2220 (2000). https://doi.org/10.1364/A0.39.002210

[6] H. Grahn, P. Geladi, Techniques and Applications of Hyperspectral Image Analysis. Wiley (2007). https://doi.org/10.1002/9780470010884

[7] J. Kerekes. "Imaging spectrometers go commercial," Laser focus world, 42(7) (2006).

[8] N. Gat, "Imaging spectroscopy using tunable filters: a review," Proc. SPIE 4056 (2000).

[9] P. García-Allende, O. Conde, J. López-Higuera. Hyperspectral imaging for raw material sorting and processed product quality control. In Applications and Experiences of Quality Control. Intech (2011). https://doi.org/10.5772/15740

[10] X. Prieto-Blanco, C. Montero-Orille, B. Couce, and R. de la Fuente. Optical configurations for imaging spectrometers. In Intelligence for Remote Sensing, volume 133, Springer Berlin/Heidelberg (2008). https://doi.org/10.1007/978-3-540-79353-3_1

[11] P. Mouroulis, M. McKerns. "Pushbroom imaging spectrometer with high spectroscopic data fidelity: experimental demonstration," Opt. Eng., 39(3), 808-816 (2000). https://doi.org/10.1117/1.602431

[12] W. L. Wolfe. Introduction to Imaging Spectrometers. SPIE Press, (1997). https://doi.org/10.1117/3.263530

[13] J. S. Pearlman, P. S. Barry, C. C. Segal, J. Shepanski, D. Beiso, S. L. Carman. "Hyperion, a space-based imaging spectrometer," IEEE Trans. on Geoscience and Remote Sensing, 41(6), 1160-1173 (2003). https://doi.org/10.1109/TGRS.2003.815018

[14] R. L. Lucke, M. Corson, N. R. McGlothlin, S. D. Butcher, D. L. Wood et al. "Hyperspectral imager for the coastal ocean: instrument description and first images," Appl. Opt. 50, 1501-1516 (2011) https://doi.org/10.1364/A0.50.001501

[15] X. Prieto-Blanco, C. Montero-Orille, B. Couce, and R. de la Fuente. "Analytical design of an Offner imaging spectrometer," Optics Express, 14(20), 9156-9168 (2006). https://doi.org/10.1364/OE.14.009156

[16] X. Prieto-Blanco, C. Montero-Orille, H. González-Núñez, M. D. Mouriz, E. López-Lago, R. de la Fuente. "The Offner imaging spectrometer in quadrature," Optics Express, 18(12), 12756-12769 (2010). https://doi.org/10.1364/OE.18.012756

[17] P. Mouroulis, B. van Gorp, R. O. Green, D. Cohen, D. Wilson et al. "Design of an airborne portable remote imaging spectrometer (PRISM) for the coastal ocean," Pasadena, CA: JPL (2010).

[18] C. Montero-Orille, X. Prieto-Blanco, H. González-Núñez, R. de la Fuente, "Two-wavelength anastigmatic Dyson imaging spectrometers," Opt. Lett., 35(14), 2379-2381, (2010). https://doi.org/10.1364/OL.35.002379

[19] E. Herrala, J. T. Okkonen, T. S. Hyvarinen, M. Aikio, and J. Lammasniemi, "Imaging spectrometer for process industry applications," Proc. SPIE 2248, 33-40 (1994). https://doi.org/10.1117/12.194344

[20] C. Yang, J. H. Everitt, M. R. Davis, C. Mao. "A CCD camera-based hyperspectral imaging system for stationary and airborne applications," Geocarto International, 18(2), 71-80 (2003). https://doi.org/10.1080/10106040308542274

[21] M. Goto, S. Morita, "Spatial distribution measurement of atomic radiation with an astigmatism-corrected Czerny-Turner spectrometer in the Large Helical Device," Rev. Sci. Instrum. 77 (2006). https://doi.org/10.1063/1.2216987

[22] D. R. Austin, T. Witting, I. A. Walmsley, "Broadband astigmatism-free Czerny-Turner imaging spectrometer using spherical mirrors," Appl. Opt., 48(19), 3846-3853 (2009). https://doi.org/10.1364/A0.48.003846

[23] D. Bannon. "Information across the spectrum," SPIE Oemagazine (2004).

[24] J. Fisher, M. M. Baumback, J. H. Bowles, J. M. Grossmann, J. A. Antoniades. "Comparison of low-cost hyperspectral sensors," Proc. SPIE 3438, (1998). https://doi.org/10.1117/12.328112

[25] C. Montero-Orille, X. Prieto-Blanco, H. González-Núñez, R. de la Fuente, "Design of Dyson imaging spectrometers based on the Rowland circle concept," Appl. Opt. 50, 6487-6494 (2011). https://doi.org/10.1364/A0.50.006487 
[26] M. D. Mouriz, E. López Lago, X. Prieto-Blanco, H. González-Núñez, R. de la Fuente, "Schwarzschild spectrometer," Appl. Opt. 50, 2418-2424 (2011). https://doi.org/10.1364/A0.50.002418

[27] X. Prieto-Blanco, H. González-Nuñez, R. de la Fuente, "Off-plane anastigmatic imaging in Offner spectrometers," J. Opt. Soc. Am. A 28, 2332-2339 (2011). https://doi.org/10.1364/JOSAA.28.002332

[28] H. González-Núñez, X. Prieto-Blanco, R. de la Fuente, "Pupil aberrations in Offner spectrometers," J. Opt. Soc. Am. A 29, 442-449 (2012). https://doi.org/10.1364/JOSAA.29.000442

[29] H. González-Núñez, “Espectrómetros de imagen Offner,” Tesis doctoral, http://hdl.handle.net/10347/9797

[30] R. L. Lucke. "Out-of-plane dispersion in an Offner spectrometer," Opt. Eng., 46(7), 073004, (2007).

[31] X. Prieto-Blanco, D. Mouriz, H. González-Núñez, E. López-Lago, R. de la Fuente,"Differential ray tracing analysis of the Schwarzschild objective,". Proc. SPIE 8001 (2011). https://doi.org/10.1117/12.892018

[32] J. M. Howard, B. D. Stone, "Imaging a point with two spherical mirrors," J. Opt. Soc. Am. A 15 (1998). https://doi.org/10.1364/josaa.15.003045

[33] H. A. Buchdahl. An Introduction to Hamiltonian Optics. Dover Publications (1993).

[34] B. D. Stone, G. W. Forbes, "Second-order design methods for definitive studies of plane-symmetric, twomirror systems," J. Opt. Soc. Am. A, 11(12), 3292 (1994). https://doi.org/10.1364/JOSAA.11.003292

[35] X. Prieto-Blanco, C. Montero-Orille, H. González-Núñez, M. Mouriz, E. López, R. de la Fuente, "Imaging with classical spherical diffraction gratings: the quadrature configuration," J. Opt. Soc. Am. A 26 (2009). https://doi.org/10.1364/JOSAA.26.002400

[36] H. G. Beutler, "The Theory of the Concave Grating," J. Opt. Soc. Am. 35, 311-350 (1945). https://doi.org/10.1364/JOSA.35.000311

[37] P. Mouroulis, "Optical design and engineering: lessons learned," Proc. SPIE 5865 (2005).

[38] H. González-Núñez, C. Vázquez, E. Lopez Lago, M. Mouriz, C. Montero-Orille, X. Prieto-Blanco, R. de la Fuente, "Design, calibration and assembly of an Offner imaging spectrometer," JPCF 274 (2011).

[39] H. González-Núñez, R. de la Fuente, "White light interferometry applied to wavelength calibration of spectrometers," Proc. SPIE 8785 (2013). https://doi.org/10.1117/12.2026187

\section{Introducción}

Una fotografía RGB convencional ofrece información acerca de una escena en tres bandas espectrales anchas proporcionando una información espectral limitada para cada punto de la escena. La espectrometría de imagen, también conocida por el nombre de espectroscopía de imagen o por el término inglés hyperspectral imaging entre otros, se define como la adquisición de imágenes en cientos de bandas espectrales contiguas de forma que el espectro de radiación de cada píxel de la imagen es registrado [1]. Por contraposición con la espectrometría convencional el espectro de cada punto de una escena se graba de forma independiente. Este espectro, representa la fracción de la energía que es reflejada o emitida por un punto de la imagen en función de la longitud de onda. En la figura 1 se muestra una representación de una imagen hiperespectral adquirida en nuestro laboratorio con uno de los sensores hiperespectrales diseñado, ensamblado y caracterizado durante la tesis doctoral.

Durante años, la espectrometría de imagen ha estado ligada a la teledetección terrestre y astronómica y las primeras aplicaciones científicas de los sensores hiperespectrales fueron geológicas, ecológicas, estudios costeros, cartografiado, amenazas ambientales, agricultura, silvicultura, estudios urbanos... [1-5]. Los avances en el diseño de nuevos prototipos, las mejoras en los algoritmos de procesado de datos hiperespectrales, el desarrollo de modelos de reflectancia espectral y la aparición de métodos semiempíricos de recuperación de parámetros de la superficie de un material [4], junto con la mayor capacidad de computación y la aparición de detectores comerciales de bajo coste, han permitido la incursión de la espectrometría de imagen en nuevos campos y aplicaciones donde esta tecnología resulta útil. De este modo, la espectrometría de imagen también se utiliza en medicina, biología, control de procesos, seguridad, colorimetría o conservación del patrimonio entre otros [6-9]. A pesar de todos estos 
avances, uno de los principales inconvenientes de la utilización de esta tecnología en nuevos campos sigue siendo el elevado coste de un equipo hiperespectral de alta calidad. Esto se debe a que la tecnología desarrollada para el diseño y fabricación de estos equipos ha estado principalmente motivada por aplicaciones militares o aeroespaciales, donde los elevadísimos costes de producción de un único prototipo son asumibles. En este contexto, la finalidad de este proyecto de tesis ha sido el diseño, montaje y análisis de espectrómetros de imágenes de bajo coste, autónomos y precisos, que permitan extraer y analizar la información espectral de una escena y que puedan ser utilizados en diferentes aplicaciones.

(a) Representación del cubo de datos:

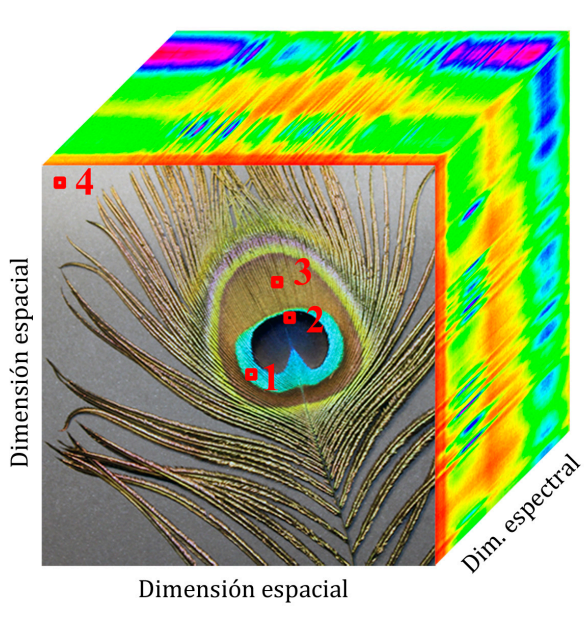

(b) Espectro de diferentes píxeles espaciales:
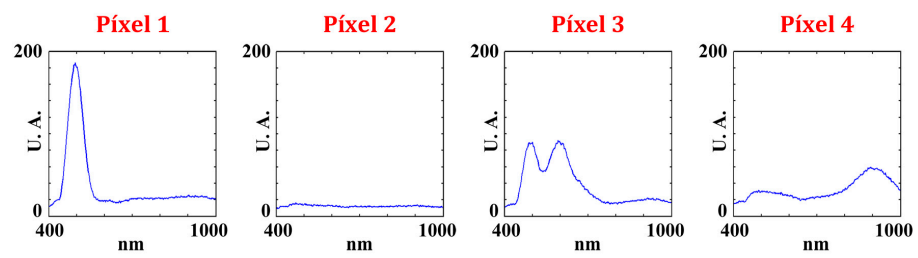

(c) Imágenes en diferentes longitudes de onda:
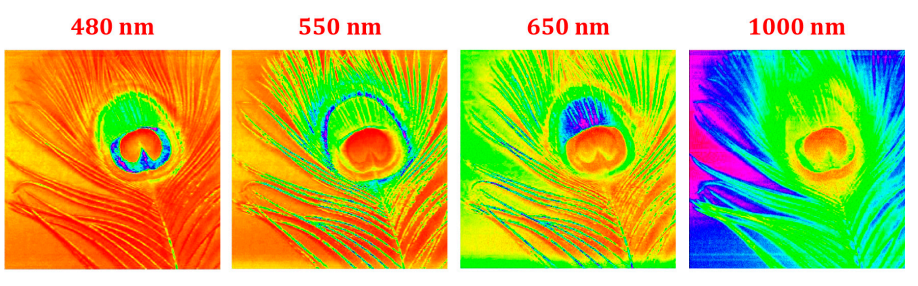

Fig.1. Representación de una imagen hiperespectral adquirida con uno de los prototipos montado en el laboratorio durante la tesis doctoral en el rango 400-1000 nm. (a) Representación en forma de cubo de datos con dos dimensiones espaciales y una espectral. (b) Espectro de diferentes píxeles de la imagen. (c) Imágenes en diferentes longitudes de onda.

\section{1.a. Espectrómetros de imagen dispersivos tipo pushbroom: Sistema Offner}

Existen diferentes técnicas para la adquisición de imágenes hiperespectrales con diferentes métodos de adquisición de una imagen del cubo de datos (whiskbroom, pushbroom, framing o windowing) y con diferentes procedimientos de obtención de la información espectral (elementos dispersivos, sistemas basados en filtros o espectrómetros por transformada de Fourier) [10]. Este trabajo se ha centrado en el desarrollo de espectrómetros de imagen dispersivos tipo pushbroom. Un espectrómetro de imagen dispersivo separa la radiación electromagnética en diferentes ángulos en función de su longitud de onda con la ayuda de prismas, redes de difracción o una combinación de ambos. En un sistema dispersivo tipo pushbroom, la imagen de una línea de una escena es dispersada y focalizada sobre una matriz de detectores bidimensional. Las columnas de la matriz de detectores (paralelas a la dirección de la línea) contienen la información espacial mientras que la información espectral se encuentra en las filas, pudiendo considerarse cada fila como un espectrómetro independiente. La otra dimensión espacial es adquirida con un sistema de barrido de forma que la escena es procesada línea por línea. La calibración estos sensores presenta serias dificultades y algunos de los artefactos que pueden aparecer en este tipo de sensores, llevan a la producción de firmas espectrales falsas [11]. El sistema dispersivo forma, en el plano imagen, diferentes imágenes monocromáticas de la rendija de entrada desplazadas lateralmente. Idealmente, las imágenes de la rendija son líneas rectas y el aumento lateral de la rendija en cada longitud de onda es el mismo. En un sistema real, sin embargo, las imágenes no son totalmente rectas y presentan cierta curvatura, fenómeno conocido como smile. Por otra parte, el aumento lateral de la misma puede variar en función de la longitud de onda apareciendo la distorsión conocida como keystone. A pesar de estas complicaciones, los sensores dispersivos tipo pushbroom, sin necesidad de partes móviles, alcanzan mayores relaciones SNR (Signal-to-Noise Ratio) que aquellos que escanean la escena punto a punto, obteniendo el espectro de un único punto en cada adquisición (sensor tipo whiskbroom) y son elegidos frente a estos en diversas aplicaciones como, por ejemplo, la teledetección desde satélites en órbita $[5,12]$.

Un sensor hiperespectral dispersivo tipo pushbroom tiene tres componentes fundamentales: una óptica externa, un sistema dispersivo y una unidad de detección (ver fig. 2). La óptica externa forma imagen de una escena sobre la rendija de entrada del sistema dispersivo que separa el espectro de la luz proveniente 
de dicha rendija en ángulos diferentes en función de su longitud de onda en una dirección perpendicular a la misma y forma imagen sobre la unidad de detención. Entre algunos de los sistemas estudiados y utilizados como unidad dispersiva en un sensor hiperespectral tipo pushbroom, se pueden destacar el sistema Offner [13-16], el sistema Dyson [17, 18], los sistemas PGP (Prism-Grating-Prism) [19, 20] o modificaciones del sistema Czerny-Turner [21, 22].

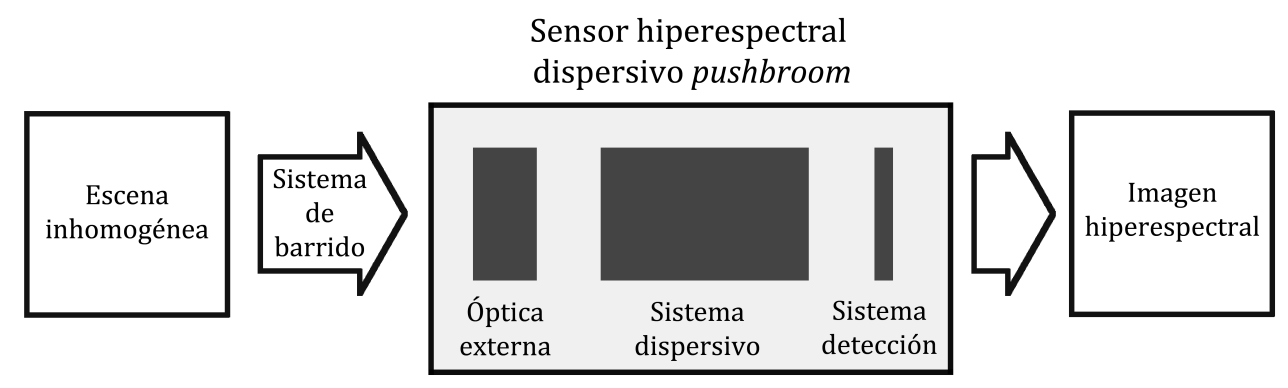

Fig.2. Diagrama de un sensor hiperespectral dispersivo pushbroom con sus tres componentes fundamentales: óptica externa, sistema dispersivo y sistema de detección.

El espectrómetro Offner (ver fig. 3) esta compuesto por dos o tres superficies reflectantes esféricas situadas concéntricamente. La principal diferencia con el espectrómetro Czerny-turner es la utilización de una red de difracción convexa. Esta configuración tiene la ventaja de tener una gran calidad de imagen y baja distorsión para aperturas numéricas altas. La posibilidad de construir sistemas simples, compactos, con altas aperturas numéricas y de fácil alineamiento con muy buena calidad de imagen, hace que hoy en día sea uno de los sistemas más utilizados en espectrometría de imagen tanto de altas prestaciones como en sistemas de bajo coste $[11,15,23,24]$.

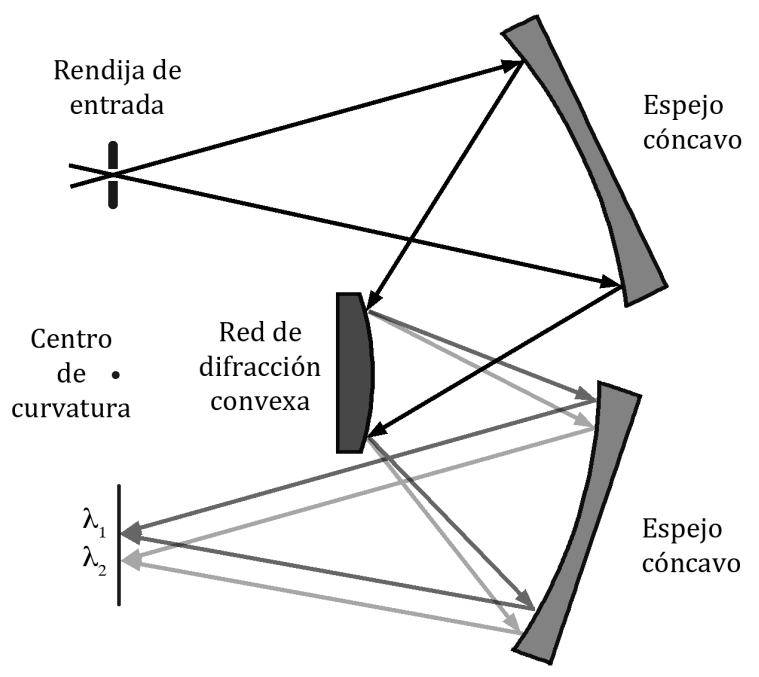

Fig. 3. Esquema de la unidad espectral de un sensor hiperespectral Offner.

La aparición en el mercado de detectores bidimensionales comerciales de alta calidad, junto con las características que ofrecen los sensores dispersivos tipo pushsbroom motivó el interés por el estudio y análisis de sistemas de separación espectral basados en configuraciones concéntricas como es el sistema Offner, con el objetivo de diseñar espectrómetros de imagen con mejores prestaciones manteniendo un reducido coste en su fabricación. Para ello, en este trabajo se han analizado sistemas que utilizan únicamente elementos esféricos con redes de difracción clásicas (no corregidas en aberraciones) y con un número de elementos lo más reducido posible. En concreto la investigación se ha centrado en el sistema Offner aunque también se ha extendido a otras configuraciones concéntricas como el sistema Dyson [18, 25] o el sistema Schwarzschild [26].

A pesar de la aparente sencillez del sistema Offner, es fundamental una buena estrategia de diseño para obtener una buena calidad de imagen. Los programas de diseño óptico actuales mejoran la calidad de 
imagen introduciendo nuevos elementos, utilizando elementos asféricos o corregidos en aberraciones y proponiendo descentramientos o inclinaciones. Esto aumenta la complejidad y el coste del sistema y complica su alineamiento. Sin embargo, con un buen diseño analítico, basado en un profundo estudio de las aberraciones del sistema, en ocasiones es posible encontrar un punto de partida óptimo para la construcción de un espectrómetro de calidad en su forma más simple [15, 16, 27, 28].

Para resumir, los objetivos de este trabajo han sido por un lado verificar experimentalmente la validez de las estrategias de diseño desarrolladas y por otro lado el desarrollo de un protocolo con las técnicas de alineamiento y caracterización necesarias para la construcción de prototipos de laboratorio de espectrómetros de imagen dispersivos tipo pushbroom basados en el sistema Offner. Para ello, en la sección 2 se presenta una breve introducción de los fundamentos teóricos, modelos y técnicas analíticas utilizadas para el diseño de espectrómetros de imagen concéntricos y se presentan los diseños de dos unidades de dispersión basadas en el sistema Offner. En la sección 3 se explican los procedimientos necesarios para el alineamiento y montaje de estos dos prototipos y en la sección 4 se presentan brevemente los resultados de la caracterización de ambos prototipos obtenidos con diversas técnicas de caracterización desarrolladas también durante este proyecto de tesis. Por último, la sección 5 incluye las conclusiones generales del trabajo realizado. Para un análisis más detallado de los temas tratados en cada una de estas secciones, se remite al lector al texto completo de la tesis doctoral [29].

\section{Diseño óptico}

Actualmente, los diseñadores ópticos tienen a su disposición herramientas computacionales como lenguajes de programación, hojas de cálculo y en general multitud de software especializado. A pesar de los grandes avances en este campo, el principal problema del diseño óptico es la no linealidad de las funciones utilizadas para describir un sistema con cambios en las propias variables que lo definen. Una de las formas más conocidas para linealizar el problema es reducir las relaciones trigonométricas exactas para el camino descrito por un punto del frente de ondas, denominado rayo de luz, al límite cuando los ángulos y las alturas involucrados tienden a cero. Para un sistema centrado, con ciertas simetrías en torno a un eje llamado eje óptico, este procedimiento es conocido como óptica paraxial y estos términos son suficientes para describir la posición y el tamaño de la imagen. Sin embargo, en aquellos sistemas en los que para la formación de imagen se utilizan regiones de los elementos fuera de la zona paraxial (ángulos de incidencia en la superficie grandes), como el objetivo Schwarzschild o el propio sistema Offner, esta aproximación paraxial pierde su sentido [15, 30,31].

El método de diseño analítico propuesto en este trabajo parte del desarrollo en serie de Taylor del camino óptico o light path function (LPF) en torno a los parámetros de la trayectoria de un rayo real, denominado rayo base [32] y definido como el rayo que atraviesa el sistema en el centro del cono de rayos que sale del punto objeto y que alcanzan el punto imagen, es decir, el rayo real que atraviesa el sistema por el centro del diafragma de apertura. El análisis directo de la trayectoria de este rayo base a través del desarrollo en serie del mismo [31, 33, 34], permite obtener información sobre la posición y las características de la imagen de forma analítica y exacta. En la óptica de sistemas centrados este rayo sería el propio eje óptico, y se estaría realizando un estudio parabasal análogo a los estudios paraxiales en torno a dicho eje. El procedimiento utilizado, consiste en anular sucesivamente los diferentes órdenes en el desarrollo en serie de Taylor de la LPF. Al anular el primer orden, se obtiene la dirección del rayo base. Anulando el segundo orden, se obtiene la condición de imagen. Los órdenes superiores están relacionados respectivamente con las diferentes aberraciones de orden superior. Con esta técnica, el Principio de Huygens-Fresnel en el límite de longitudes de onda pequeñas o equivalentemente el Principio de Fermat es utilizado directamente para localizar la posición y determinar las características de la imagen de forma analítica. Este método de diseño parte, al igual que la óptica paraxial o de primer orden, del punto de vista de la óptica Hamiltoniana [33, 34], en la que una de las funciones características asociada a un sistema óptico se expande en serie de Taylor alrededor de un rayo particular. En la mayoría de sistemas ópticos, las expresiones obtenidas con este método resultan demasiado complicadas pero, en determinados sistemas se obtienen expresiones analíticas relativamente sencillas y manejables. De esta forma, teniendo en cuenta las ligaduras correspondientes al sistema óptico de estudio y escogiendo los parámetros adecuados, es posible imponer unas condiciones de formación de imagen particulares y obtener un buen diseño, cancelando determinadas aberraciones. El principal objetivo de este método analítico es la obtención de un buen punto de partida teórico para un determinado sistema óptico en su forma más simple. En un paso 
posterior, partiendo de dicho punto, se podrían llevar a cabo pequeños refinamientos realizando una optimización por métodos numéricos.

El sistema Offner, es un sistema extremadamente simple con un número muy reducido de elementos, compacto y de fácil alineamiento. Explorar mediante un método analítico las configuraciones óptimas y optimizar la calidad del sistema en su forma más simple, permite la construcción de sistemas más económicos que aquellos que introducen elementos adicionales, redes corregidas o superficies no esféricas. En este sistema (fig. 3), la rendija de entrada debe situarse bastante alejada del centro de curvatura del sistema para evitar el viñeteo que produce la red de difracción de forma que las teorías de aberraciones de Seidel o Buchdahl no resultan ser las más apropiadas debido a los grandes ángulos de incidencia en las superficies. Sin embargo, un estudio parabasal habitualmente usado en el estudio de aberraciones en redes de difracción cóncavas [35,36], permite obtener expresiones analíticas simples sin restricciones en los ángulos de incidencia que pueden ser aplicadas directamente en el diseño de espectrómetros de imagen de este tipo [15, 16, 27, 28]. Diferentes estudios se han llevado a cabo aplicando este procedimiento buscando la eliminación o minimización de determinadas aberraciones de bajo orden como son el astigmatismo y el coma. En algunos estudios se han analizando las posiciones de las imágenes intermedias creadas por cada elemento $[15,16]$ o, equivalentemente, se ha asumido una iluminación astigmática sobre la red de difracción [27]. Sin embargo, también es posible realizar un análisis parabasal tomando el espectrómetro Offner como un sistema completo, procedimiento publicado en González-Núnez et al. [28]. A continuación, se resumen las principales conclusiones de este estudio.

La expresión exacta para la LPF de un espectrómetro de imagen Offner ha sido determinada y se ha demostrado que la trayectoria de cualquier rayo en el espacio objeto e imagen sólo depende de un parámetro, relacionado con la distancia al centro de curvatura de dicho rayo en cada espacio. Con el análisis de la LPF completa se ha conseguido, por tanto, estudiar las propiedades de formación de imagen para posiciones objeto e imagen arbitrarias evitando el análisis, habitualmente más complicado, de las imágenes intermedias formadas por cada superficie. La expansión en serie de potencias de la LPF en las coordenadas de la red permite analizar las aberraciones del orden deseado para cualquier ángulo de incidencia del rayo principal y facilita la búsqueda de configuraciones con aberraciones de bajo orden reducidas. Aunque las expresiones resultantes son en general bastante complejas, los casos particulares en los que estas expresiones son simples son identificados fácilmente. Estos casos corresponden con los denominados Rowland mounts [28], donde se verifica la reducida presencia de aberraciones de bajo orden (astigmatismo y coma), en concordancia con la literatura existente. El análisis realizado ha demostrado que, además del astigmatismo, todos los términos de tercer orden en el desarrollo en serie de potencias se pueden cancelar en la imagen del punto de diseño para una longitud de onda cuando dicho punto se encuentra en el plano que contiene al centro de curvatura de las superficies esféricas y que es perpendicular a la línea que une dicho centro de curvatura con el centro de la red de difracción esférica que actúa como diafragma de apertura del sistema [28]. En esta configuración, las aberraciones relevantes están asociadas con los términos de cuarto orden de la expansión. Estos términos (relacionados con aberraciones esféricas de orden superior entre otras) han sido calculados y se ha deducido numéricamente que las aberraciones de orden superior tienen un peso insignificante siendo los términos de cuarto orden los que representan la aberración predominante del sistema. Finalmente, en este estudio también se han analizado los términos de tercer y cuarto orden de la expansión para Rowland mounts con el punto de diseño fuera de dicho plano. En esta situación uno de los términos de tercer orden no se cancela y esta aberración compite con las aberraciones asociadas a los términos de cuarto orden. De todos modos, es importante destacar que en todas las configuraciones analizadas, las aberraciones se mantienen reducidas para números f bajos a pesar de los grandes ángulos de incidencia necesarios para evitar el viñeteo que puede producir la red de difracción. El smile y keystone también se mantienen muy bajos, quedando típicamente reducidos a una pequeña fracción del píxel.

Con estas técnicas y procedimientos se han realizado dos diseños. Un espectrómetro de imagen en el rango 400-1000 nm (VIS-NIR) basado en un sistema Offner en plano y un sistema en el rango 530-660 nm (VIS) basado en un sistema Offner fuera de plano. Parámetros como rango espectral, dispersión o tamaño de la rendija de entrada, entre otros, han sido escogidos teniendo en cuenta valores de referencia de equipos hiperespectrales diseñados o publicados anteriormente o por razones como tamaño, sensibilidad espectral de la matriz de detectores o coste. Las simulaciones numéricas llevadas a cabo para implementar los resultados de los estudios analíticos y para verificar su calidad han sido realizadas en su totalidad en una distribución gratuita de software de diseño óptico denominada OSLO-EDU (Lambda Research Corp.). 
La técnica utilizada en el diseño del Espectrómetro de imagen Offner VIS-NIR está basada en un trabajo previo desarrollado por el grupo de investigación [15]. Las expresiones teóricas utilizadas en dicho trabajo anulan, en una configuración en plano, el astigmatismo y su derivada espectral para un punto de la rendija de entrada y para una longitud de onda, de forma que para longitudes de onda cercanas a la de diseño, el astigmatismo y el coma se mantienen reducidos. Las características del diseño realizado se encuentran en la tabla 1 . Se han escogido el tamaño y el rango espectral típicos de un sensor CCD de 2/3" (8.8 x 6.6 mm y 400-1000 nm), una red de difracción con un número de líneas por milímetro estándar de 150 líneas/mm y un número f de 2.4, teniendo en consideración la obtención de un equipo con un tamaño manejable. El valor de los radios de curvatura de las 3 superficies se indica en la tabla 1 con el valor del parámetro $\mathrm{R}_{\mathrm{i}}$. Este diseño presenta buenas características: alta resolución y bajas distorsiones resaltando que no se utiliza ningún elemento asférico ni redes corregidas en aberraciones mientras que la conservación de la concentridad contribuye a facilitar el alineamiento. Finalmente destacar que el peor dato de RMS obtenido es de $12.1 \mu \mathrm{m}$ para los extremos de la rendija de entrada en la longitud de onda de $1000 \mathrm{~nm}$ y que el smile y keystone quedan reducidos a $0.1 \mu \mathrm{m}$ y $0.3 \mu \mathrm{m}$ respectivamente.

El diseño del sistema hiperespectral Offner VIS 530-660 nm está basado en la configuración analizada en la publicación Prieto-Blanco et al. [16]. En esta configuración, en la que el centro de la rendija de entrada del espectrómetro se sitúa lejos del plano de dispersión y denominada en cuadratura, se ha pretendido obtener un sistema de calidad con un único espejo cóncavo en lugar de los dos utilizados habitualmente. Los resultados de las simulaciones (tabla 1) muestran un sistema con una excelente resolución espacialespectral, un RMS menor de $5.6 \mu \mathrm{m}$ y unas distorsiones muy reducidas: un smile menor que $0.04 \mu \mathrm{m}$ y un keystone menor que $0.08 \mu \mathrm{m}$; utilizando sólo un espejo cóncavo y una red de difracción clásica.

En vista de los resultados obtenidos en ambos diseños, se puede afirmar que las técnicas empleadas no sólo permiten diseñar rápidamente espectrómetros tipo Offner más simples y con calidades de imagen comparables a otros diseños, generalmente numéricos y que utilizan elementos ya corregidos $[15,16]$. Al mismo tiempo han permitido analizar y explorar nuevas configuraciones en las que la rendija de entrada del espectrómetro se encuentra fuera del plano de dispersión [27, 28]. Finalmente cabe destacar la utilidad de estas técnicas de diseño que, además del sistema Offner, han sido aplicadas con éxito en otros sistemas concéntricos formadores de imagen [31] y de espectrometría de imagen [18, 25, 26].

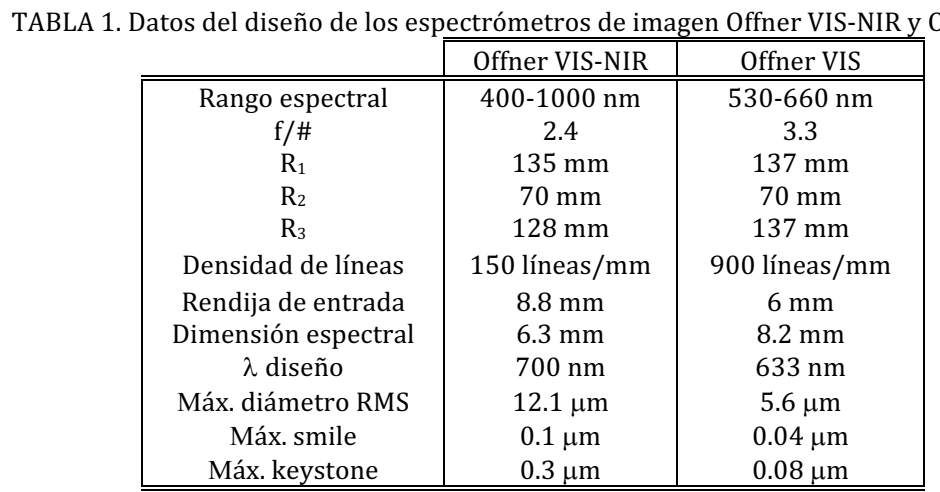

\section{Alineamiento Óptico}

Una vez que las técnicas de diseño fueron expuestas y corroboradas numéricamente, en esta sección se presenta el material utilizado y los pasos realizados para construir y alinear dos prototipos de laboratorio de espectrómetros de imagen tipo Offner basados en los diseños presentados en la sección anterior. Es necesario remarcar que por muy bueno que sea un diseño, es totalmente inútil sin un plan de montaje y alineamiento viable y no extremadamente complejo. Sobre todo teniendo en cuenta que el alineamiento de un sistema con espejos fuera de eje como es el sistema Offner es especialmente crítico [37].

Todo el material óptico y optomecánico necesario para el montaje de los prototipos, incluyendo las redes de difracción convexas, ha sido adquirido de distribuidores comerciales de material óptico de laboratorio. Debido a las características singulares impuestas por el diseño teórico en valores como radios de curvatura o diámetros de apertura, los espejos esféricos, la red de difracción y las rendijas de entrada han sido fabricados bajo demanda. Por otra parte, determinadas piezas como los soportes de los espejos, de la 
red de difracción o del detector, han sido diseñadas y fabricadas en el propio laboratorio. En la tabla 2 se presentan los principales datos de los componentes utilizados en ambos prototipos. El protocolo de alineamiento completo está descrito en el capítulo 3 de la tesis doctoral [29]. A continuación se incluye una breve descripción con las etapas más importantes del mismo.

TABLA 2 Datos de los componentes utilizados en cada uno de los dos prototipos.

\begin{tabular}{|c|c|c|}
\hline & Offner VIS-NIR & Offner VIS \\
\hline Óptica externa & \multicolumn{2}{|c|}{ Schneider Xenoplan 1.4/23mm Compact } \\
\hline Rendija de entrada & $15 \mu \mathrm{m} \times 10 \mathrm{~mm}$ & $10 \mu \mathrm{m} \times 10 \mathrm{~mm}$ \\
\hline $\begin{array}{c}\text { Espejos cóncavos: } \\
\text { Radio de curvatura } \\
\text { Diámetro de apertura } \\
\end{array}$ & $\begin{array}{c}135 \mathrm{~mm} \text { y } 128 \mathrm{~mm} \\
65 \mathrm{~mm} \text { y } 65 \mathrm{~mm}\end{array}$ & $\begin{array}{l}137 \mathrm{~mm} \\
105 \mathrm{~mm}\end{array}$ \\
\hline $\begin{array}{l}\text { Red de difracción: } \\
\text { Radio de curvatura } \\
\text { Diámetro de apertura } \\
\text { Líneas por milímetro }\end{array}$ & $\begin{array}{r}70 \mathrm{~mm} \\
29 \mathrm{~mm} \\
150 \mathrm{l} / \mathrm{mm} \\
\end{array}$ & $\begin{array}{r}70 \mathrm{~mm} \\
21 \mathrm{~mm} \\
900 \mathrm{l} / \mathrm{mm} \\
\end{array}$ \\
\hline $\begin{array}{l}\text { Sistema de detección: } \\
\text { Tamaño del sensor } \\
\text { Tamaño de píxel } \\
\end{array}$ & \multicolumn{2}{|c|}{$\begin{array}{c}\text { CCD QImaging Retiga } 2000 \mathrm{R} 1600 \times 1200 \\
11.84 \mathrm{~mm} \times 8.88 \mathrm{~mm} \\
7.4 \mu \mathrm{m} \times 7.4 \mu \mathrm{m} \\
\end{array}$} \\
\hline
\end{tabular}

La principal característica del sistema Offner, y el punto más importante en su alineamiento, es la disposición concéntrica de las superficies esféricas de las que consta. Un láser de He-Ne y una lámina de desplazamiento lateral (shear-plate) son utilizados para alcanzar la concentricidad de forma interferométrica. Partiendo de un haz colimado, un sistema convergente se utiliza para focalizar el haz sobre una red de difracción plana auxiliar que ilumina el/los espejos esféricos con dos de sus órdenes de difracción. El orden cero de la red es utilizado para asegurar el posicionamiento del foco del sistema convergente sobre la superficie de dicha red. Esto sucede cuando el haz reflejado en el orden cero vuelve a emerger colimado al pasar por el sistema convergente. A continuación, los espejos cóncavos se colocan utilizando los primeros órdenes de difracción de la red plana auxiliar. La luz reflejada por los espejos de cada orden de difracción vuelve a la red y sólo cuando el centro de curvatura de espejo se sitúe en la posición del foco del sistema convergente, el haz volverá a salir colimado y en la misma dirección que el haz inicial. Por último, la red de difracción convexa se posiciona con el haz convergente que viene directamente del sistema convergente. Un punto importante es que dicha red debe ser el diafragma de apertura del sistema. Para esto, la red convexa se coloca en una plataforma de rotación de modo que su centro coincide con el centro de curvatura de la red, lo que permite la rotación de la misma sin perder la concentricidad. El centro de la rendija de entrada del espectrómetro se coloca utilizando una plataforma de traslación micrométrica desde el centro de curvatura de las superficies a su posición final. La rendija de entrada también se sitúa en sobre un sistema de rotación para ajustar la orientación de la misma asegurando que la dirección espectral y la dimensión espacial sean perpendiculares. Por último, para situar la CCD se busca la posición de la imagen de la rendija cuando esta se ilumina con un láser de He-Ne. A continuación, la rendija se ilumina con lámparas espectrales de Ar y Hg. Estas lámparas son elegidas porque cubren todo el rango espectral de ambos sistemas. En esta última etapa, la CCD es ligeramente desplazada de nuevo para encontrar una posición de alta calidad espacial y espectral.

La técnica descrita permite alinear de forma completa cualquiera de los diseños de espectrómetros de imagen tipo Offner realizados, tanto configuraciones con uno como con dos espejos cóncavos. El procedimiento completo de alineamiento es algo laborioso pero siguiendo el protocolo detallado minuciosamente en el capítulo 3 de la tesis [29] se puede realizar todo el proceso de alineado en menos de 4 horas, una vez que el material necesario se encuentra preparado. 


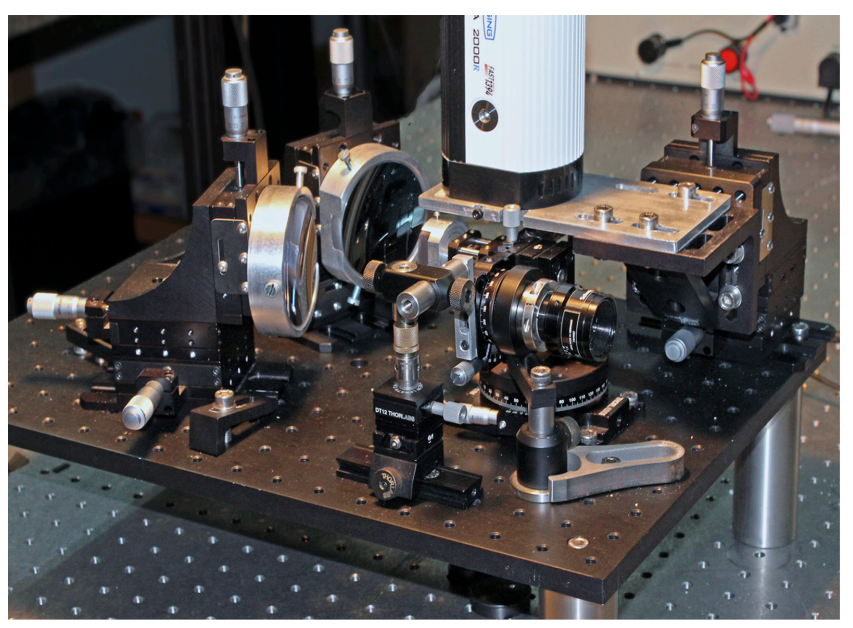

(a)

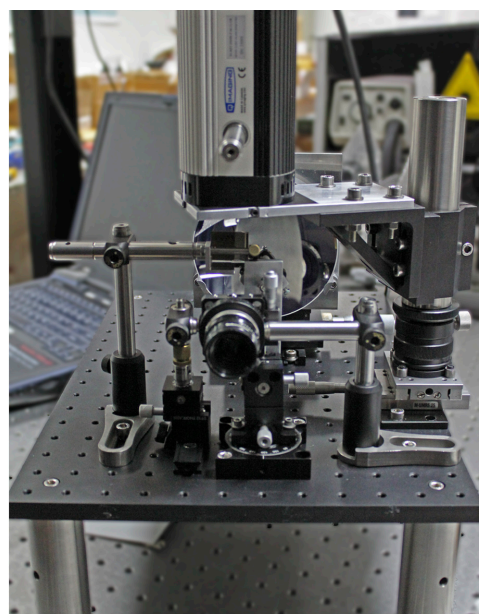

(b)

Fig.4. Fotografías de los dos prototipos totalmente funcionales construidos durante el proyecto de tesis. (a) Espectrómetro de imagen VIS de 400-1000 nm. (b) Espectrómetro de imagen VIS de 530-660 nm.

\section{Caracterización y calibración de prototipos}

El objetivo de esta sección ha sido analizar de la calidad de los prototipos montados en el laboratorio utilizando el procedimiento brevemente descrito en la sección anterior. Diferentes técnicas de calibración y caracterización han sido analizadas y nuevos métodos han sido propuestos [38, 39]. El trabajo se ha centrado principalmente en realizar una buena calibración espectral, la medida de la resolución espectral y espacial y en una caracterización exhaustiva de las dos principales distorsiones presentes en este tipo de sistemas: smile y keystone. Los resultados se presentan resumidos en la tabla 3.

TABLA 3. Principales resultados de la caracterización de los prototipos VIS-NIR y VIS. El tamaño de un píxel (píx.) corresponde a $7.4 \mu \mathrm{m}$. (*) Datos correspondientes a Binning 4.

\begin{tabular}{|c|c|c|}
\hline & Offner VIS-NIR & Offner VIS \\
\hline Rango spectral (nm) & $392.1-1002.4$ & $529.8-659.8$ \\
\hline Dispersión $(\mathrm{nm} / \mu \mathrm{m})$ & 0.0959 & 0.0160 \\
\hline № bandas espaciales * & 298 & 203 \\
\hline № bandas espectrales * & 215 & 277 \\
\hline FWHM espacial (píx.) & $\leq 4.84$ & $\leq 3.80$ \\
\hline FWHM espectral (píx.) & $\leq 4.17$ & $\leq 1.78$ \\
\hline Smile (píx.) & $\leq 0.6$ & $\leq 0.30$ \\
\hline Keystone (píx.) & $\leq 1.6$ & $\leq 0.38$ \\
\hline $\mathrm{FOV}\left({ }^{0}\right)$ & 21.7 & 15.1 \\
\hline $\operatorname{IFOV}\left({ }^{0}\right)^{*}$ & 0.074 & 0.074 \\
\hline
\end{tabular}

Las técnicas presentadas en el capítulo 4 de la tesis doctoral [29] y no incluidas aquí, han resultado ser efectivas para la caracterización óptica de ambos prototipos y pueden ser aplicadas para cualquier sistema hiperespectral dispersivo tipo pushbroom. Los resultados corroboran la calidad espacial y espectral de ambos prototipos. También se ha verificado que los principales factores que limitan la calidad óptica de ambos instrumentos no son debidos al diseño de la unidad dispersiva. Los factores que más contribuyen a la pérdida de resolución y/o al aumento de distorsiones son: los defectos de la rendija de entrada, que puede ser un factor limitante en la resolución espectral pero que en ambos casos influye principalmente en la distorsión a lo largo de la dimensión espacial; la red de difracción convexa que al no tener un blaze optimizado resulta poco eficiente y debido a esto y a su calidad puede introducir luz parásita en el detector (otros órdenes, reflejos o scattering) y la óptica externa refractiva que puede afectar a la resolución espacial y a la distorsión en la dimensión espacial. Otro factor a tener en cuenta en es el detector que, además de limitar la resolución espacial y espectral del sistema, especialmente cuando se utiliza con binning 4, resulta un factor limitante en la caracterización debido a la frecuencia de muestreo del mismo. Por último, las tolerancias, tanto en las incertidumbres en el valor de los parámetros de los elementos ópticos utilizados como en posibles errores en el alineamiento, pueden contribuir a explicar las ligeras diferencias entre los resultados experimentales y los obtenidos por simulación. 


\section{Conclusiones}

A lo largo de este proyecto de tesis doctoral se han desarrollado las técnicas de diseño, los métodos de montaje, alineamiento y caracterización necesarios para la construcción de prototipos de laboratorio de espectrómetros de imagen Offner. En concreto, dichas técnicas han sido utilizadas para diseñar, ensamblar y caracterizar dos prototipos: un sistema hiperespectral en el rango 400-1000 $\mathrm{nm}$ basado en un sistema Offner en plano y otro en el rango 530-660 $\mathrm{nm}$ basado en un sistema Offner fuera de plano.

En el capítulo 1 de esta tesis se ha justificado la necesidad del desarrollo de sensores hiperespectrales de alta calidad y bajo coste para su aplicación en actividades con un presupuesto reducido y se ha propuesto el sistema Offner como un sistema que cumple ambos requisitos. En el capítulo 2 se han presentado técnicas de diseño que parten de modelos totalmente analíticos basados en la teoría de aberración de redes y que, aplicadas al sistema Offner, han permitido optimizar el sistema en su forma más simple por medio de la eliminación o minimización de determinadas aberraciones de bajo orden como son el astigmatismo y el coma. Las técnicas desarrolladas son eficientes para diseñar rápidamente espectrómetros tipo Offner con calidades de imagen comparables a otros diseños, generalmente numéricos, que utilizan elementos no esféricos o redes de difracción corregidas en aberraciones. Al mismo tiempo, estas técnicas han permitido analizar y explorar nuevas configuraciones en las que la rendija de entrada del espectrómetro se encuentra fuera del plano de dispersión. En este mismo capítulo, se han presentado dos diseños particulares de espectrómetros de imagen Offner realizados siguiendo dichas técnicas y su calidad ha sido verificada numéricamente con la ayuda de una versión gratuita de un software comercial de diseño óptico. En el capítulo 3 se han descrito los elementos ópticos y optomecánicos utilizados junto con un relativamente sencillo y rápido protocolo de alineamiento desarrollado durante este proyecto de tesis que, explotando la configuración concéntrica del sistema Offner, ofrece un método que permite alinear un sistema hiperespectral Offner completo en unas pocas horas. En el capítulo 4 se presentan las técnicas de caracterización desarrolladas para evaluar la calidad de cualquier sistema hiperespectral dispersivo tipo pushbroom. Estas técnicas han resultado ser efectivas para la caracterización óptica de ambos prototipos. La principal conclusión del análisis de los resultados llevado a cabo en este capítulo y por tanto del trabajo realizado en este proyecto de tesis, ha sido la verificación experimental de las técnicas de diseño analíticas expuestas en el capítulo 2 y la validación de los métodos de alineamiento expuestos en el capítulo 3 que permiten la construcción de prototipos de espectrómetros de imagen tipo Offner de alta calidad.

A partir del trabajo realizado en esta tesis, se deduce que el principal y único inconveniente para la obtención de un espectrómetro de imagen Offner de alta calidad y bajo coste es la utilización de una red de difracción convexa. Una red de alta calidad con un blaze optimizado eleva el coste del sistema de forma considerable debido únicamente a la escasa comercialización de redes de difracción convexas. Por último vale la pena mencionar que, como continuación de este trabajo y en base a los satisfactorios resultados obtenidos, resultaría útil para numerosas aplicaciones la construcción de equipos portátiles y autónomos a partir de los prototipos de laboratorio ya construidos y validados.

\section{Agradecimientos}

Los trabajos de investigación llevados a cabo durante esta tesis doctoral han sido realizados fundamentalmente en el marco de los proyectos de investigación: Shiper: Sistema hiperespectral aplicado a sensorización medioambiental. Óptica e montaxe (07MDS035166PR) y Opto-Sensing. Consolidación e estructuración de unidades de investigación competitivas (CN 2012/156) financiados por la Xunta de Galicia. El autor también agradece la concesión de un contrato predoctoral del Programa María Barbeito 2009 financiado por la Xunta de Galicia que disfrutó entre los años 2010 y 2012 y del contrato postdoctoral FONDECYT № 3150343. 